\title{
Revista

\section{Consumo e custo de recursos materiais em unidades pediátricas de terapia intensiva e semi-intensiva}

\author{
Costs and consumption of material resources in pediatric intensive and semi-intensive care units
}

Costo y consumo de recursos materiales en unidades pediátricas de terapia intensiva y semi-intensiva

\section{Larissa Lenotti Zuliani', Marli de Carvalho Jericó", Liliana Cristina de Castro'"', Zaida Aurora Sperli Geraldes Soler"V}

' Faculdade de Medicina de São José do Rio Preto, Curso de Enfermagem (Graduanda). São José do Rio Preto-SP, Brasil.

"Faculdade de Medicina de São José do Rio Preto, Departamento de Enfermagem Especializada. São José do Rio Preto-SP, Brasil.

"' Universidade de São Paulo, Escola de Enfermagem, Programa de Pós-Graduação em Gerenciamento em Enfermagem e em Saúde (Doutoranda). São Paulo-SP, Brasil.

IV Faculdade de Medicina de São José do Rio Preto, Departamento de Enfermagem em Saúde Coletiva e Orientação Profissional, Programa de Pós-Graduação em Enfermagem. São José do Rio Preto-SP, Brasil.

\section{Submissão: 23-08-2010 Aprovação: 22-01-2013}

\section{RESUMO}

O gerenciamento de custos de materiais de consumo hospitalar é um tema atual em pesquisa, principalmente em unidades de saúde especializadas. Os enfermeiros são destacados como os principais gestores do consumo e custo de materiais hospitalares. Neste estudo, objetivou-se caracterizar unidades pediátricas semi-intensivas e intensivas de um hospital de ensino e verificar o consumo e os custos de materiais utilizados na assistência a pacientes internados nessas unidades. Estudo descritivo, exploratório, retrospectivo, com abordagem quantitativa; os dados foram obtidos do Sistema de Informação Hospitalar; analisados com base na classificação ABC. O gasto médio foi semelhante entre as UTIs cardiológica e neonatal e menor nas UTI e semi-intensiva pediátricas; houve variação significativa de consumo mensal de materiais; os materiais de maior custo tiveram mais impacto no orçamento das unidades estudadas. Os dados obtidos revelaram a importância do uso de método sistêmico de análise de consumo e gastos de materiais em unidades pediátricas e subsidiam ações administrativas de economia.

Descritores: Economia da Enfermagem; Custos Hospitalares; Administração Hospitalar; Unidades de Terapia Intensiva; Pediatria.

\section{ABSTRACT}

Cost management of hospital material resources is a trendy research topic, especially in specialized health units. Nurses are pointed out as the main managers for costs and consumption of hospital materials resources. This study aimed to characterize Pediatric Intensive and Semi-Intensive Care Units of a teaching hospital and investigate costs and consumption of material resources used to treat patients admitted to these units. This is a descriptive exploratory study with retrospective data and quantitative approach. Data were obtained from a Hospital Information System and analyzed according to the ABC classification. The average expenditures were similar in both the neonatal and cardiac units, and lower in Pediatric Intensive and Semi-Intensive care units. There was a significant variation in the monthly consumption of materials. Higher cost materials had a greater impact on the budget of the studied units. The data revealed the importance of using a systematic method for the analysis of materials consumption and expenditure in pediatric units. They subsidize administrative and economic actions.

Key words: Economics Nursing; Hospital Costs; Hospital Administration; Intensive Care Units; Pediatrics.

\section{RESUMEN}

La gestión de costos de materiales de consumo hospitalario es un tema actual en investigación, especialmente en unidades de salud especializadas. Los enfermeros son destacados como los principales gestores de consumo y costos de materiales hospitalarios. En este estudio, objetivó-se caracterizar Unidades Pediátricas de Terapia Intensiva (UTI) y Semi-intensiva de un hospital escuela e investigar el consumo y costo de materiales utilizados en la asistencia de pacientes ingresados en esas unidades. Estudio descriptivo, exploratorio, retrospectivo, de abordaje cuantitativo. Los datos fueron obtenidos del Sistema de Información Hospitalaria y analizados de acuerdo con la clasificación ABC. El gasto medio fue semejante entre las UTIs cardiológica y neonatal, y menor en las unidades de terapia intensiva y semi-intensiva pediátricas. Hubo variación significativa en el consumo mensual de materiales. Los materiales de mayor costo presentaron un mayor impacto en el presupuesto de las unidades estudiadas. Los datos revelan la importancia del uso de un método sistémico de análisis de consumo y gastos de materiales en unidades pediátricas y subsidian acciones administrativas de economía.

Palabras clave: Economía de la Enfermería; Costos de Hospital; Administración Hospitalaria; Unidades de Cuidados Intensivos; Pediatría. 


\section{INTRODUÇÃO}

A crise política e econômica mundial dos últimos anos também repercutiu no Brasil e tornou a gestão de serviços de saúde um desafio ainda maior. Os avanços da tecnologia e de insumos na indústria farmacêutica e de materiais e de equipamentos geraram aumento dos gastos, insuficiência de recursos e dificuldades de controle. Os enfermeiros têm se destacado nas ações gerenciais de unidades de saúde e uma de suas preocupações tem sido a administração racional de recursos materiais, articulando-se com vários segmentos da sociedade, com vistas a alcançar melhor qualidade de atendimento e maior produtividade para as empresas de assistência em saúde ${ }^{(1)}$.

O desafio na gestão de custos em saúde é adotar medidas racionalizadoras, para equilibrar a qualidade dos serviços prestados com as limitações orçamentárias, que são frequentes $^{(1-4)}$. Então, além do quadro de pessoal, que abrange majoritariamente profissionais de enfermagem, os medicamentos e materiais são os recursos que mais elevam os custos hospitalares, com consumo estimado entre 15 a $45 \%$ do orçamento de tais organizações ${ }^{(2)}$.

O gerenciamento de custos na assistência em saúde é um processo administrativo de tomada de decisão, na busca de eficiente racionalização na alocação de recursos disponíveis, respeitando tanto as necessidades da clientela quanto as finalidades institucionais ${ }^{(1)}$. A equipe de enfermagem é a maior usuária de recursos materiais na assistência em saúde e a atuação gerencial do enfermeiro na contenção de gastos envolve várias medidas, entre outras: estilo de liderança, processo de comunicação que é estabelecido com os funcionários, satisfação no trabalho, adequação do espaço físico, dos recursos matérias e do dimensionamento de pessoal ${ }^{(1,3,5)}$.

Na gestão de custos em saúde, é recomendado a aplicação de métodos sistêmicos, como o método $A B C$, que preconiza o agrupamento dos itens consumidos em classes, segundo faixa de participação no orçamento. A Classe A representa cerca de $20 \%$ dos itens que correspondem a quase $50 \%$ do custo; a Classe C representa $50 \%$ do total de itens e aproximadamente $20 \%$ do custo; e os $30 \%$ de itens restantes pertencem à Classe $B{ }^{(1,3)}$. A classificação $A B C$ facilita o gerenciamento dos estoques nas organizações, sendo também perfeitamente aplicável na gestão de materiais e insumos usados em instituições de saúde, permitindo evidenciar os materiais prioritários ${ }^{(3)}$.

As Unidades de Terapia Intensiva Pediátricas estão entre as principais consumidoras do orçamento hospitalar, em decorrência de suas peculiaridades assistenciais, das tecnologias, materiais e equipamentos que utilizam para promover uma assistência qualificada para alcance de melhores resultados na atenção a crianças em estado crítico ${ }^{(6)}$.

Diante das considerações estabelecidas, os objetivos desta pesquisa foram: caracterizar unidades pediátricas semi-intensivas e intensivas de um hospital de ensino e verificar o consumo e os custos de materiais utilizados na assistência a pacientes internados nessas unidades.

\section{MÉTODOS}

\section{Delineamento}

Trata-se de um estudo descritivo - exploratório, com coleta de dados realizada em unidades pediátricas de Terapia Intensiva (UTI) e semi-intensiva (USI) de um hospital de ensino de capacidade extra, situado no interior do Estado de São Paulo. Essa instituição presta assistência hospitalar e ambulatorial em várias especialidades da área da saúde, com média de 2.500 internações mensais e 2.000 cirurgias/mês de pequeno, médio e grande porte.

Antecedendo a coleta de dados, o projeto deste estudo foi autorizado na instituição hospitalar e depois aprovado por Comitê de Ética em Pesquisa em seres humanos (Parecer CEP $n^{\circ}$ 151/2009). O Sistema de Tecnologia da Informação (STI) do hospital disponibilizou dados para caracterizar as unidades pediátricas pesquisadas (perfil demográfico e clínico dos pacientes) e sobre o consumo de matérias usados na assistência de enfermagem e médica. Já o Centro de Educação Permanente (CEP) forneceu dados sobre a composição quali-quantitativa da equipe de enfermagem.

Vale esclarecer que nessa instituição os custos estão organizados por centro de custos e forma-se um centro de custo para cada UTI e USI juntas. O consumo de materiais foi analisado segundo as variáveis: descrição do produto, quantidade consumida, valor total na moeda (Real) e porcentagem (\%) que cada item representou sobre o custo anual, com essa variável calculada a partir do gasto total anual com materiais de cada unidade.

O procedimento empregado para realizar a classificação $A B C$ iniciou-se com a construção de uma planilha no programa Microsoft Excel $2003^{\circledR}$, selecionando-se os dez materiais mais consumidos e os de maior custo utilizados no período de coleta dos dados, em cada centro de custo, sendo posteriormente classificados segundo a curva $\mathrm{ABC}$.

\section{Análise dos Dados}

Seguiu-se as etapas descritas por Paterno ${ }^{(7)}$ na elaboração da curva e definição dos itens ABC: 1. Calculou-se os valores globais de consumo para cada item de compra no período considerado. O valor global foi resultado da multiplicação do custo unitário do produto pelo número de unidades consumidas ao longo do período; 2 . Os valores dos artigos foram colocados em ordem decrescente; 3. Calculou-se o valor acumulado despendido somando-se os valores globais de cada item, anotando os valores após a adição de cada parcela, até se obter o valor total consumido; 4. Calculou-se o valor percentual de gasto de cada item dividindo-se o seu gasto pelo total de recursos gastos; 5 . Conforme realizado no item 3, efetuou-se o cálculo de percentagens acumuladas.

\section{RESULTADOS E DISCUSSÃO}

\section{Caracterização das unidades e perfil dos pacientes}

No hospital campo deste estudo a organização dos leitos para atendimento de crianças que exigiam assistência mais complexa, seguia cuidados progressivos, com base na 
legislação profissional vigente, possibilitando a adequação entre a capacidade instalada e a condição clínica da criança ${ }^{(8)}$. De acordo com a necessidade de cuidados, as unidades de atendimento eram classificadas em Unidade de Terapia Intensiva (UTI) ou Unidade Semi - intensiva (USI).

Eram disponibilizados 44 leitos de terapia intensiva (UTI) e semi-intensiva (USI), distribuídos da seguinte forma: 20 leitos para área Neonatal, 10 leitos pediátricos e 14 leitos para área cardiológica. Em estudo realizado em 85 UTIs pediátricas e/ou neonatais do município de São Paulo, encontrou-se uma média de 18,75 leitos de UTI neonatal, 14,3 leitos de UTI pediátrica e 8,5 leitos destinados a crianças de UTI especializada ${ }^{(9)}$.

A caracterização das Unidades de Terapia Intensiva (UTI) e Semi-intensiva (USI) pediátricas incluídas nesta pesquisa e o perfil das crianças atendidas nestas unidades encontra-se descrita no Quadro 1, destacando-se nos resultados obtidos, no período de um ano de coleta dos dados:

$\checkmark$ A capacidade instalada era de 44 leitos, com atendimento prestado a crianças desde o período neonatal até a adolescência, correspondendo a 14 leitos cardiológicos, 20 leitos na área neonatal e 10 leitos pediátricos gerais;

$\checkmark$ O dimensionamento da equipe de enfermagem por leito foi de 0,2 enfermeiros e 1,8 técnicos de enfermagem na unidade cardiológica e igual número - 0,3 enfermeiros e 2,0 técnicos de enfermagem nas unidades neonatal e pediátrica. O total de enfermeiros atuantes nas três UTIs e USIs do hospital estudado era 13.
O dimensionamento de pessoal, na definição da proporção entre categoria profissionais de enfermagem e número de leitos, na composição da equipe para atuação em UTIs, tem sido tema de debates no âmbito do Ministério da Saúde - MS, do Conselho Federal de Enfermagem (COFEN), das sociedades médicas e de enfermagem. A Resolução n. ${ }^{\circ}$ 203/2004 do COFEN $^{(13)}$ trata do dimensionamento de pessoal e recomenda a distribuição percentual para cuidados semi-intensivos em $42 \%$ a $46 \%$ e intensivos de $52 \%$ a $56 \%$ de enfermeiros e o restante de técnicos de enfermagem. Nos achados desse estudo é difícil estabelecer comparações nestas categorias profissionais, uma vez que o quadro de pessoal de enfermagem é único para as UTI semi-intensivas e intensivas.

Tendo por referência normas preconizadas pelo COFEN, evidenciou-se que nas três unidades de atenção complexa a crianças, o quantitativo de enfermeiros era bem reduzido correspondendo a $3(10,7 \%)$ - na UTI e USI cardiológicas, $6(12,8 \%)$ na UTI e USI neonatal e $4(15,4 \%)$ na pediátrica.

O Ministério da Saúde ${ }^{(14)}$ preconiza para UTIs um enfermeiro assistencial para cada dez leitos e de um técnico de enfermagem para cada dois leitos por turno de trabalho. Neste estudo constatou-se que a proporção de técnicos de enfermagem nas três unidades estudadas estava de acordo com o recomendado, com 25 técnicos de enfermagem para 14 leitos de UTI e USI neonatal; 41 técnicos de enfermagem para 20 leitos de UTI e USI cardiológica e 22 técnicos de enfermagem para 10leitos de UTI e USI pediátrica. Isto evidencia que na instituição em estudo o investimento em pessoas está direcionado para o nível operacional.

\begin{tabular}{|c|c|c|c|}
\hline UNIDADES & UTI e USI CARDIOLÓGICA - N=14 & UTI E USI NEONATAL N=20 & UTI e USI PEDIÁTRICA N=10 \\
\hline Leitos & $10(\mathrm{UTI})$ e 4 (USI) & 10 (UTI) e 10 (USI) & 6 (UTI) e 4 (USI) \\
\hline População & Infantil - 0 a 12 anos & Neonatal - 0 a 28 dias & Pediátrica - 29 dias a 12 anos \\
\hline $\begin{array}{l}\text { Relação } \\
\text { Enferm/Leito }\end{array}$ & $\begin{array}{l}\text { Enfermeiro - 6- } \\
\text { Técnico Enfermagem - } 25-1,8 / 1\end{array}$ & $\begin{array}{l}\text { Enfermeiro - } 4 \\
\text { Técnico Enfermagem - } 41\end{array}$ & $\begin{array}{l}\text { Enfermeiro - } 4 \\
\text { Técnico Enfermagem - } 22-2,0 / l\end{array}$ \\
\hline $\begin{array}{l}\text { Perfil } \\
\text { Atendimentos }\end{array}$ & 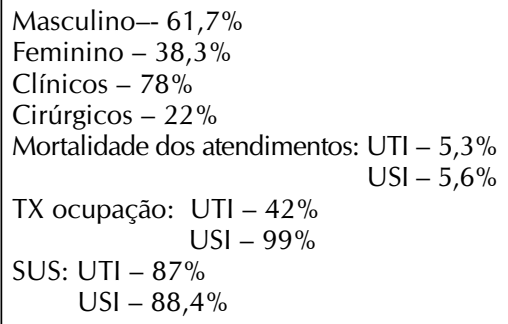 & 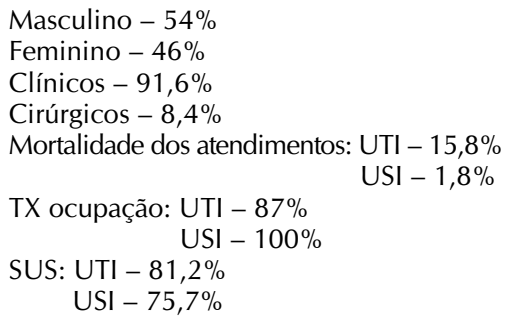 & 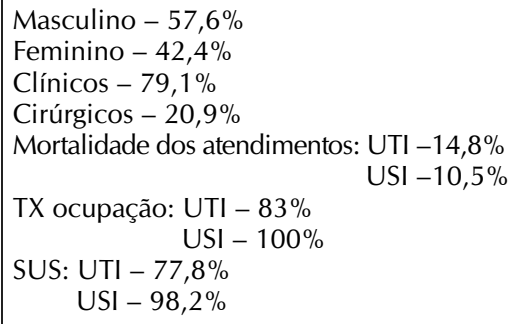 \\
\hline $\begin{array}{l}\text { Principais } \\
\text { Diagnósticos }\end{array}$ & $\begin{array}{l}\text { CIV }-13,0 \% \\
\text { Tetralogia de Fallot }-8,9 \% \\
\text { Malformação do coração }-5,8 \% \\
\text { Comunicação atrioventricular }-5 \% \\
\text { Ventrículo com dupla via }-4,6 \% \\
\text { Síndrome do choque tóxico }-4,4 \% \\
\text { Coartação da aorta }-4,1 \% \\
\text { CIA - 3,3\% } \\
\text { Comunicação ventrículo-atrial - 2,6\% } \\
\text { Insuficiência respiratória - } 2,1 \%\end{array}$ & $\begin{array}{l}\text { RN de pré-termo - } 42,4 \% \\
\text { Síndrome da angústia respiratória }-27,5 \% \\
\text { Permeabilidade do canal arterial }-2,8 \% \\
\text { Desconfortos respiratórios }-1,7 \% \\
\text { Doenças inflamatórias do SNC - } 1,6 \% \\
\text { Pneumotórax }-1,3 \% \\
\text { Pneumonia - } 1,2 \% \\
\text { Imaturidade extrema - 0,9\% } \\
\text { Espinha bífida lombar - 0,8\% } \\
\text { Infecção - 0,8\% }\end{array}$ & $\begin{array}{l}\text { Distrofia muscular - } 11,1 \% \\
\text { Doenças inflamatórias do SNC - 8,7\% } \\
\text { IRA - } 8,5 \% \\
\text { Desnutrição protéico-calórica grave - } 6,1 \% \\
\text { Desnutrição protéico-calórica } \\
\text { não especificada - 5,5\% } \\
\text { Insuficiência respiratória } \\
\text { não especificada - 5,5\% } \\
\text { Pneumonia - 3,4\% } \\
\text { Refluxo gastroesofágico - 3,1\% } \\
\text { Toxoplasmose - } 3 \% \\
\text { Leucemia }-2,7 \%\end{array}$ \\
\hline
\end{tabular}

TX - Taxa; SUS - Sistema Único de Saúde; CIA - Comunicação Interatrial; CIV - Comunicação Interventricular; RN - Recém-nascido; SNC - Sistema Nervoso Central; IRA - Insuficiência Respiratória Aguda.

Quadro 1 - Caracterização das unidades UTI e USI Cardiológica, Neonatal e Pediátrica. São José do Rio Preto, 2007 a 2008. 
Vale lembrar que uma adequada quantificação de profissionais de enfermagem deve considerar não só a proporção com número de leitos, mas especialmente a carga de trabalho associada ao processo de cuidar nessas unidades. A participação do enfermeiro nos procedimentos de alta complexidade e na coordenação do cuidado é de fundamental importância para a excelência no cuidado e cumprimento da legislação profissional em vigor $^{(15)}$.

$\checkmark$ Mais de $50 \%$ das internações foram de crianças do sexo masculino $(61,7 \%$ nas unidades cardiológicas, $54 \%$ na neonatal e $57,6 \%$ na pediátrica geral);

$\checkmark$ A maior parte das crianças tinha problemas clínicos, correspondendo a $78 \%$ na área cardiológica, 91,6\% na área neonatal e $79,1 \%$ na área pediátrica geral;

$\checkmark$ A mortalidade foi de aproximadamente $15 \%$ nas três unidades de UTI (15,3\% na cardiológica, 15,8\% na neonatal e $14,8 \%$ na pediátrica). Nas unidades semi-intensivas a taxa de mortalidade variou de 10,5\% na pediátrica, de 5,6\% na cardiológica e $1,8 \%$ na neonatal;

$\checkmark$ A taxa de ocupação nas unidades de terapia intensiva foi superior a $80 \%$ nas UTIs neonatal $(87 \%)$ e pediátrica $(83 \%)$ e de $42 \%$ na UTI cardiológica. Já a taxa de ocupação nas USI foi total nas unidades neonatal e pediátrica e de $99 \%$ na cardiológica;

$\checkmark$ Os pacientes pediátricos do SUS ocuparam mais de $70 \%$ dos 44 leitos de UTIs e USIs estudados;

$\checkmark$ Os principais diagnósticos das crianças internadas nas UTIs e USIs deste estudo foram CIV (13\%) na unidade cardiológica; prematuridade $(42,4 \%)$ na área neonatal e distrofia muscular $(11,1 \%)$ na área pediátrica geral.

O perfil clínico dos pacientes nas unidades investigadas foi semelhante ao verificado em outras pesquisas, como segue: em estudo realizado em unidade cardiológica, a comunicação interventricular (CIV) foi o diagnóstico mais frequente entre as cardiopatias $(41,8 \%)^{(10)}$; na UTI Neonatal do Instituto da Criança do Hospital das Clínicas da Faculdade de Medicina da Universidade de São Paulo, os diagnósticos mais comuns foram: doença da membrana hialina $(34,4 \%)$, comum no pré-termo, desconforto respiratório não classificado $(21,9 \%)$, risco infeccioso (18\%) e malformação digestiva $(14 \%)^{(11)}$; em UTI pediátrica de cinco hospitais de Porto Alegre predominaram os problemas respiratórios $(32,7 \%)^{(12)}$.

Análise do consumo de materiais nas unidades investigadas

Baseando-se na análise de classificação $A B C$, verifica-se na Tabela 1 que, como esperado, os materiais mais consumidos foram os de classe $C$, estocados em almoxarifado e farmácia satélite. A maior parte dos materiais foi de copos descartáveis; seringas e agulhas (nas unidades neonatal 341.128-50,9\%, na UTI e USI cardiológica 230.001-34,3\% e nas unidades pediátricas $98.725-14,7 \%$ ). As unidades de maior complexidade assistencial apresentaram um alto consumo destes materiais. Embora o consumo tenha sido semelhante àquele das unidades pediátricas $(84,75 \% / 50,3 \%)$; o custo foi significativamente menor. Na UTI e USI cardiológica, o consumo foi $77,5 \%$ e o custo representou $16,7 \%$; na UTI neonatal foi $73,8 \%$, com um custo de 19,3\%.

Entende-se a utilização dos materiais de classe C, como seringas e agulhas, pois são exigidos na administração de medicamentos a crianças, em diferentes horários, a cada dia. Em pediatria e neonatologia é freqüente a diluição ou rediluição de medicamentos devido a sua alta concentração, bem como potenciais efeitos locais e sistêmicos de certos medicamentos, podendo causar flebites, arritmias cardíacas, hipotensão e anafilaxia(16).

Também, é comum o ajuste da dosagem, pois a indústria farmacêutica não disponibiliza a maioria das drogas de uso parenteral em formulação e apresentação à faixa etária pediátrica. As seringas de 3 e $5 \mathrm{ml}$ são muito utilizadas nas unidades investigadas, por permitir uma graduação mais precisa das doses. Algumas drogas são preparadas nestas seringas e posteriormente transferidas para seringas de $10 \mathrm{ou} 20 \mathrm{ml}$ para serem utilizadas em bombas de infusão do tipo "seringa". Vale esclarecer que o uso da bomba de infusão já está incorporado no processo de cuidar nas unidades de UTI e USI no hospital estudado, pois cerca de $98 \%$ das crianças recebiam medicação intravenosa em bomba de infusão, de forma contínua ou intermitente.

O preparo e a administração de medicamentos constituem processos que integram o sistema de medicação, sendo atividade rotineira e de responsabilidade da equipe de enfermagem para o cumprimento terapêutico ${ }^{(17)}$. Salienta-se que nas unidades estudadas, o número de profissionais de enfermagem atuantes era superior ao preconizado pela legislação ${ }^{(8)}$. Também era adequado o quantitativo de equipamento disponível para infusão contínua e controlada de fluidos - "bombas de infusão" do tipo volumétrica e de seringa para uso na Terapia Intravenosa (TIV) e nutricional. A nutrição parenteral é um procedimento essencial nessas UTIs e em especial muito utilizado na área neonatal, devido ao perfil do recém-nascido(18), requerendo qualidade dos cateteres empregados, precisão na infusão, treinamento e capacitação dos profissionais que assegurem eficiência na utilização dessa terapia nutricional( ${ }^{(19)}$.

Na UTI e USI Neonatal, tendo por base o protocolo de uso de Cateter Venoso Central de Inserção Periférica (PICC), todas as medicações são administradas em seringas de $10 \mathrm{ml}$. As agulhas 40×12 e 25x08 são utilizadas no preparo e diluição de medicamentos e na transferência de soluções de uma seringa para outra.

Os materiais pertencentes à Classe $\mathrm{A}$, para a análise dos custos, segundo a classificação $\mathrm{ABC}$ são apresentados na Tabela 2. Os insumos listados representaram um impacto de $40 \%$, $45 \%$ e $76,1 \%$ nos orçamentos das UTI e USI Cardiológica, Neonatal e Pediátrica, representando respectivamente $24,2 \%, 15 \%$ e $72,1 \%$ do total dos materiais utilizados. Ficaram em destaque nos dados obtidos: o alto o custo com TIV, 9,7\% na UTI e USI Cardiológica, 14,45 na Pediátrica e $19 \%$ na Neonatal; utilização tecnológica nos diferentes tipos de cateteres intravenosos periféricos e centrais (curta e longa permanência) de acordo com o perfil assistencial e terapêutico da clientela assistida em cada unidade; identificou-se que o lançamento do cateter duplo lúmen $13 \mathrm{~cm}$ e o pediátrico na 
Tabela 1 - Descrição dos dez materiais mais utilizados (classe C) nas UTI e USI Cardiológica, Neonatal e Pediátrica, segundo ordem decrescente de consumo. São José do Rio Preto, 2007 a 2008.

\begin{tabular}{|c|c|c|c|c|c|c|c|c|c|c|c|c|c|c|}
\hline \multicolumn{5}{|c|}{ UTI e USI Cardiológica } & \multicolumn{5}{|c|}{ UTI e USI Neonatal } & \multicolumn{5}{|c|}{ UTI e USI Pediátrica } \\
\hline \multirow{2}{*}{$\begin{array}{l}\text { Produto } \\
\text { Copo desc. } \\
\text { Água }\end{array}$} & \multicolumn{2}{|c|}{ Quantidade } & \multicolumn{2}{|c|}{ V. Total (R\$) } & \multirow{2}{*}{$\begin{array}{l}\text { Produto } \\
\text { Copo desc. } \\
\text { Água }\end{array}$} & \multicolumn{2}{|c|}{ Quantidade } & \multicolumn{2}{|c|}{ V. Total (R\$) } & \multirow{2}{*}{$\begin{array}{l}\text { Produto } \\
\begin{array}{l}\text { Seringa desc. } \\
5 \mathrm{ml}\end{array}\end{array}$} & \multicolumn{2}{|c|}{ Quantidade } & \multicolumn{2}{|c|}{ V. Total (R\$) } \\
\hline & 40700 & $13,7 \%$ & 479 & $0,4 \%$ & & 66400 & $14,4 \%$ & 777 & $0,4 \%$ & & 21142 & $18,1 \%$ & 1.820 & $6,1 \%$ \\
\hline $\begin{array}{l}\text { Seringa desc. } \\
3 \mathrm{ml}\end{array}$ & 37151 & $12,5 \%$ & 3.231 & $3,0 \%$ & $\begin{array}{l}\text { Agulha desc. } \\
25 \times 8\end{array}$ & 40230 & $8,7 \%$ & 1.596 & $0,8 \%$ & $\begin{array}{l}\text { Seringa desc. } \\
3 \mathrm{ml}\end{array}$ & 17454 & $15,0 \%$ & 1.512 & $5,0 \%$ \\
\hline $\begin{array}{l}\text { Agulha desc. } \\
25 \times 8\end{array}$ & 32456 & $10,9 \%$ & 1.290 & $1,2 \%$ & $\begin{array}{l}\text { Agulha desc. } \\
40 \times 12\end{array}$ & 40221 & $8,7 \%$ & 2.377 & $1,1 \%$ & $\begin{array}{l}\text { Seringa desc. } \\
20 \mathrm{ml}\end{array}$ & 13976 & $12,0 \%$ & 3.477 & $11,6 \%$ \\
\hline $\begin{array}{l}\text { Agulha desc. } \\
40 \times 12\end{array}$ & 32374 & $10,9 \%$ & 1.904 & $1,8 \%$ & $\begin{array}{l}\text { Seringa desc. } \\
5 \mathrm{ml}\end{array}$ & 39068 & $8,5 \%$ & 3.368 & $1,6 \%$ & $\begin{array}{l}\text { Agulha desc. } \\
25 \times 8\end{array}$ & 9063 & $7,8 \%$ & 360 & $1,2 \%$ \\
\hline $\begin{array}{l}\text { Seringa desc. } \\
5 \mathrm{ml}\end{array}$ & 26199 & $8,8 \%$ & 2.263 & $2,1 \%$ & $\begin{array}{l}\text { Seringa desc. } \\
10 \mathrm{ml}\end{array}$ & 36577 & $7,9 \%$ & 6.438 & $3,1 \%$ & $\begin{array}{l}\text { Equipo soro } \\
\text { simples }\end{array}$ & 8677 & $7,4 \%$ & 3.447 & $11,5 \%$ \\
\hline $\begin{array}{l}\text { Seringa desc. } \\
10 \mathrm{ml}\end{array}$ & 21804 & $7,4 \%$ & 3.838 & $3,6 \%$ & $\begin{array}{l}\text { Seringa desc. } \\
3 \mathrm{ml}\end{array}$ & 35549 & $7,7 \%$ & 3.091 & $1,5 \%$ & $\begin{array}{l}\text { Seringa desc. } \\
10 \mathrm{ml}\end{array}$ & 8535 & $7,3 \%$ & 1.502 & $5,0 \%$ \\
\hline $\begin{array}{l}\text { Copo desc. } \\
\text { Café }\end{array}$ & 13500 & $4,6 \%$ & 76 & $0,1 \%$ & $\begin{array}{l}\text { Copo desc. } \\
\text { Café }\end{array}$ & 24500 & $5,3 \%$ & 139 & $0,1 \%$ & $\begin{array}{l}\text { Agulha desc. } \\
40 \times 12\end{array}$ & 7015 & $6,0 \%$ & 422 & $1,4 \%$ \\
\hline $\begin{array}{l}\text { Eletrodo uti } \\
\text { adulto desc. }\end{array}$ & 10945 & $3,7 \%$ & 2.189 & $2,1 \%$ & $\begin{array}{l}\text { Seringa desc. } \\
1 \mathrm{ml}\end{array}$ & 22082 & $4,8 \%$ & 4.157 & $2,0 \%$ & $\begin{array}{l}\text { Seringa desc. } \\
1 \mathrm{ml}\end{array}$ & 6407 & $5,5 \%$ & 1.206 & $4,0 \%$ \\
\hline $\begin{array}{l}\text { Seringa desc. } \\
20 \mathrm{ml}\end{array}$ & 8868 & $3,0 \%$ & 2.244 & $2,1 \%$ & $\begin{array}{l}\text { Seringa desc. } \\
20 \mathrm{ml}\end{array}$ & 19933 & $4,3 \%$ & 5.039 & $2,4 \%$ & $\begin{array}{l}\text { Agulha desc. } \\
13 \times 4,5\end{array}$ & 3495 & $3,0 \%$ & 150 & $0,5 \%$ \\
\hline $\begin{array}{l}\text { Agulha desc. } \\
13 \times 4,5\end{array}$ & 6004 & $2,0 \%$ & 260 & $0,2 \%$ & $\begin{array}{l}\text { Tira medir } \\
\text { glicemia }\end{array}$ & 16568 & $3,6 \%$ & 12.922 & $6,2 \%$ & $\begin{array}{l}\text { Cat intrav } \\
\text { perif } 25\end{array}$ & 2961 & $2,5 \%$ & 1.188 & $4,0 \%$ \\
\hline TOTAL & 230001 & $77,5 \%$ & 17.772 & $16,7 \%$ & TOTAL & 341128 & $73,8 \%$ & 39.903 & $19,3 \%$ & TOTAL & 98725 & $84,7 \%$ & 15.084 & $50,3 \%$ \\
\hline
\end{tabular}

Desc. - descartável, Cat intrav perif - cateter intravenoso periférico.

UTI neonatal constituiu-se em erro de digitação, uma vez que esse dispositivo não é utilizado nessa unidade e sim nas UTI pediátricas e cardiológica.

A punção intravenosa periférica é um dos procedimentos invasivos mais realizados durante a hospitalização de pacientes, mesmo na área neonatal e pediátrica, para administração de fármacos e componentes sanguíneos e nutricionais ${ }^{(20)}$. A passagem do dispositivo PICC, anteriormente, restringia-se a UTI e USI Neonatal e especificamente a neonatos prematuros extremos e de muito baixo peso. Durante este estudo, apenas alguns enfermeiros eram qualificados e podiam realizar este procedimento; por direito assegurado pela Resolução COFEN 258/2001 ${ }^{(21)}$.

O dispositivo PICC, classificado como material de alto custo (classificação A), tinha acentuado controle administrativo no hospital estudado, justificando-se a restrição de seu uso pelo impacto na conta hospitalar. Atualmente, esse procedimento é realizado nas demais unidades investigadas por propiciar qualidade e segurança assistencial à criança crítica, após domínio e incorporação dessa tecnologia pelos enfermeiros atuantes nas unidades pediátricas estudadas.

Com o advento de novas terapêuticas aliadas à tecnologia, tem havido demanda crescente de novos materiais com altos custos. Conseqüentemente, é importante que os enfermeiros que atuam em unidades especializadas, analisem a necessidade de incorporação destes novos dispositivos. Para tanto devem buscar adquirir conhecimentos sobre a disponibilidade desses produtos no mercado e suas características específicas, principalmente no que diz respeito à efetividade e segurança, além do custo para a definição programática de atendimento ${ }^{(1,20)}$.

Ante tal perspectiva de análise, a comunicação efetiva entre médicos e enfermeiros é vital para a excelência na indicação do dispositivo intravenoso mais apropriado. No processo de cuidar em enfermagem grande parte das atividades se destina a pacientes recebendo algum tipo de terapia intravenosa. Em muitos países, o enfermeiro é responsável pela seleção, inserção e remoção dos cateteres venosos centrais e periféricos, bem como a avaliação, manutenção e orientações ao paciente e à família ${ }^{(20)}$.

Os tipos e custos dos equipos utilizados na Terapia Intravenosa (TIV) são vários. Neste estudo, o equipo de soro simples representou alto gasto somente nas UTI e USI pediátricas, onde havia menor complexidade assistencial e terapêutica. $\mathrm{O}$ equipo de bomba de infusão (enteral, parenteral e foto equipo), mostrou-se entre os itens de maior custo nas UTI e USI cardiológica $(2.932-7,9 \%)$, neonatal $(5.648-9,8 \%)$ e pediátrica $(1.940-18,6 \%)$. Pode-se interpretar que esse resultado é 
Tabela 2 - Descrição dos dez materiais de maior custo (classe A) utilizados nas UTI e USI Cardiológica, Neonatal e Pediátrica, segundo ordem decrescente. São José do Rio Preto, 2007 a 2008.

\begin{tabular}{|c|c|c|c|c|c|c|c|c|c|c|c|c|c|c|}
\hline \multicolumn{5}{|c|}{ UTI e USI Cardiológica } & \multicolumn{5}{|c|}{ UTI e USI Neonatal } & \multicolumn{5}{|c|}{ UTI e USI Pediátrica } \\
\hline Produto & Quan & tidade & V. To & al (R\$) & Produto & Quan & tidade & V. Tot & al (R\$) & Produto & Quan & tidade & V. Tot & al (R\$) \\
\hline $\begin{array}{l}\text { Equipo } \\
\text { bomba } \\
\text { de inf. }\end{array}$ & 2932 & $1,0 \%$ & 8.477 & $7,9 \%$ & $\begin{array}{l}\text { PICC } \\
24 / 25 \mathrm{~g}\end{array}$ & 85 & $0,0 \%$ & 16.210 & $8,5 \%$ & $\begin{array}{l}\text { Seringa } \\
\text { desc. } 20 \mathrm{ml}\end{array}$ & 13976 & $12,0 \%$ & 3.477 & $11,6 \%$ \\
\hline $\begin{array}{l}\text { CVCLP } \\
13 \mathrm{~cm}\end{array}$ & 77 & $0,0 \%$ & 6.747 & $6,3 \%$ & $\begin{array}{l}\text { Tira medir } \\
\text { glicemia }\end{array}$ & 16568 & $3,6 \%$ & 12.922 & $6,8 \%$ & $\begin{array}{l}\text { Equipo soro } \\
\text { simples }\end{array}$ & 8677 & $7,4 \%$ & 3.447 & $11,5 \%$ \\
\hline $\begin{array}{l}\text { Tira medir } \\
\text { glicemia }\end{array}$ & 5876 & $2,0 \%$ & 4.583 & $4,3 \%$ & $\begin{array}{l}\text { Equipo bomba } \\
\text { de Inf. (parent.) }\end{array}$ & 4051 & $0,9 \%$ & 11.729 & $6,2 \%$ & $\begin{array}{l}\text { Cat intrav } \\
\text { perif } 24\end{array}$ & 2959 & $2,5 \%$ & 3.116 & $10,4 \%$ \\
\hline $\begin{array}{l}\text { Seringa } \\
\text { desc. } 10 \mathrm{ml}\end{array}$ & 21804 & $7,4 \%$ & 3.838 & $3,6 \%$ & $\begin{array}{l}\text { Cat intrav } \\
\text { perif } 24\end{array}$ & 7241 & $1,6 \%$ & 7.516 & $4,0 \%$ & $\begin{array}{l}\text { Equipo } \\
\text { bomba de } \\
\text { inf. (enteral) }\end{array}$ & 1055 & $0,9 \%$ & 3.017 & $10,0 \%$ \\
\hline CVCLP $5 \mathrm{fr}$ & 64 & $0,0 \%$ & 3.661 & $3,4 \%$ & CVCLP $13 \mathrm{~cm}$ & 79 & $0,0 \%$ & 7.055 & $3,7 \%$ & $\begin{array}{l}\text { Equipo } \\
\text { bomba de } \\
\text { inf. (parent.) }\end{array}$ & 885 & $0,8 \%$ & 2.571 & $8,6 \%$ \\
\hline $\begin{array}{l}\text { Transdutor } \\
\text { de pressão }\end{array}$ & 73 & $0,0 \%$ & 3.650 & $3,4 \%$ & $\begin{array}{l}\text { Foto equipo } \\
\text { bomba de inf. }\end{array}$ & 1597 & $0,3 \%$ & 6.788 & $3,6 \%$ & $\begin{array}{l}\text { Seringa } \\
\text { desc. } 5 \mathrm{ml}\end{array}$ & 21142 & $18,1 \%$ & 1.820 & $6,1 \%$ \\
\hline $\begin{array}{l}\text { Seringa } \\
\text { desc. } 3 \mathrm{ml}\end{array}$ & 37151 & $12,5 \%$ & 3.231 & $3,0 \%$ & $\begin{array}{l}\text { Seringa } \\
\text { desc. } 10 \mathrm{ml}\end{array}$ & 36577 & $7,9 \%$ & 6.438 & $3,4 \%$ & $\begin{array}{l}\text { Seringa } \\
\text { desc. } 3 \mathrm{ml}\end{array}$ & 17454 & $15,0 \%$ & 1.512 & $5,0 \%$ \\
\hline $\begin{array}{l}\text { Extensor p/ } \\
\text { perfusão }\end{array}$ & 3838 & $1,3 \%$ & 3.119 & $2,9 \%$ & $\begin{array}{l}\text { Clorexidina } \\
\text { degermante }\end{array}$ & 626 & $0,1 \%$ & 5.832 & $3,1 \%$ & $\begin{array}{l}\text { Seringa } \\
\text { desc. } 10 \mathrm{ml}\end{array}$ & 8535 & $7,3 \%$ & 1.502 & $5,0 \%$ \\
\hline $\begin{array}{l}\text { Sensor ox. } \\
\text { de pulso }\end{array}$ & 5 & $0,0 \%$ & 2.985 & $2,8 \%$ & Papel toalha & 2475 & $0,5 \%$ & 5.496 & $2,9 \%$ & $\begin{array}{l}\text { Seringa } \\
\text { desc. } 1 \mathrm{ml}\end{array}$ & 6407 & $5,5 \%$ & 1.206 & $4,0 \%$ \\
\hline $\begin{array}{l}\text { Fr. coletor } \\
\text { móvel } 5 \text { Its. }\end{array}$ & 15 & $0,0 \%$ & 2.400 & $2,3 \%$ & $\begin{array}{l}\text { CVCLP } \\
\text { pediátrico }\end{array}$ & 84 & $0,0 \%$ & 5.352 & $2,8 \%$ & $\begin{array}{l}\text { Cat intrav } \\
\text { perif } 25\end{array}$ & 2961 & $2,5 \%$ & 1.188 & $4,0 \%$ \\
\hline TOTAL & 71835 & $24,2 \%$ & 42.689 & $40,0 \%$ & TOTAL & 69383 & $15,0 \%$ & 85.337 & $45,0 \%$ & TOTAL & 84051 & $72,1 \%$ & 22.856 & $76,1 \%$ \\
\hline
\end{tabular}

Inf.- infusão, CVCLP - Cateter Venoso Central de Longa Permanência, Ox. - oxímetro, Fr. - frasco, parent. - parenteral, Cat intrav perif - cateter intravenoso periférico, Desc. - descartável.

decorrente de decisão de uso de tecnologia que utiliza equipos específicos do fabricante da bomba de infusão.

O perfil assistencial e terapêutico da clientela atendida necessita de equipamentos mais precisos, pois podem exigir administração de decímetros de mililitros de solução a cada hora; uma vez que essas crianças têm o sistema cardiovascular menos desenvolvido causando dificuldade em adaptar-se a mudança rápida e ou grande quantidade de volume, podendo apresentar quadros de hipervolemia caso o volume administrado na TIV não seja cuidadosamente monitorado ${ }^{(20)}$.

Os recém-nascidos são os mais vulneráveis a apresentar desequilíbrio de glicemia (hipo e hiperglicemia) nos primeiros dias de vida, justificando o alto consumo (16.568/6,8\%) das tiras para medir glicemia na UTI e USI Neonatal. Após análise do impacto nos custos desse produto, houve revisão da rotina do teste glicêmico, sendo incorporado ao protocolo de manuseio mínimo do recém-nascido. Antes era aferido sistematicamente três vezes/dia e passou a ser realizado uma vez/ dia, juntamente com a coleta de exames. Percebe-se que a diminuição da frequência de venopunção, evita a manipulação excessiva dos pacientes e gastos desnecessários. Em estudo em UTI neonatal verificou-se que as respostas fisiológicas e comportamentais do recém nascido durante o manuseio e propôs protocolo de manuseio mínimo ${ }^{(22)}$.

Analisando as Tabelas 1 e 2 observa-se que os dez materiais de maior consumo (Tabela 1), não tiveram participação significativa nos orçamentos das unidades quanto os dez de maior custo (Tabela 2) - diferença média de 25,6\%. Isso ocorreu porque os materiais mais utilizados no período possuíam baixo custo.

\section{Análise do custo mensal de materiais nas unidades in- vestigadas}

Nota-se na Figura 1 que houve predominância de gastos nas UTI e USI Neonatal durante o período estudado, com gastos de R\$189,6 mil (média R\$ 15,8 mil); a UTI e USI Cardiológica acumularam um gasto de $\mathrm{R} \$ 106,6$ mil (média $\mathrm{R} \$ 8,9$ mil), e as UTI e USI Pediátrica de R\$ 30 mil (média R\$ 2,5 mil). Um dos fatores que explica tais achados diz respeito ao maior número de leitos e clientes assistidos - 6.613, ou seja, o dobro das demais unidades, como apresentado na caracterização das unidades estudadas (Quadro1). 


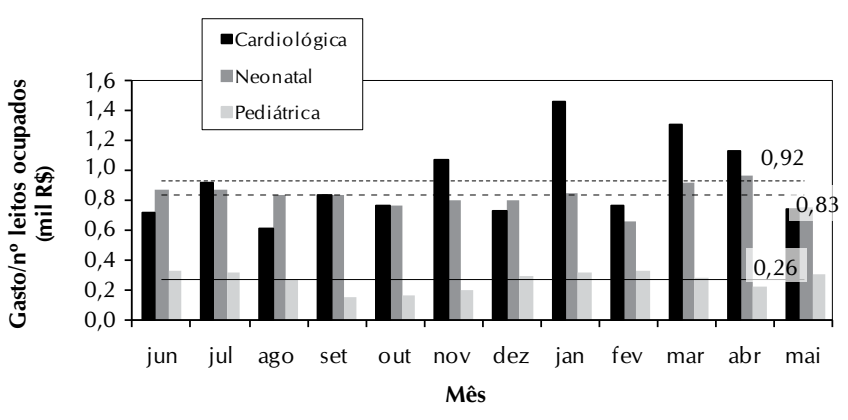

Figura 1 - Histórico mensal por unidades dos gastos com recursos materiais. São José do Rio Preto, 2007 a 2008.

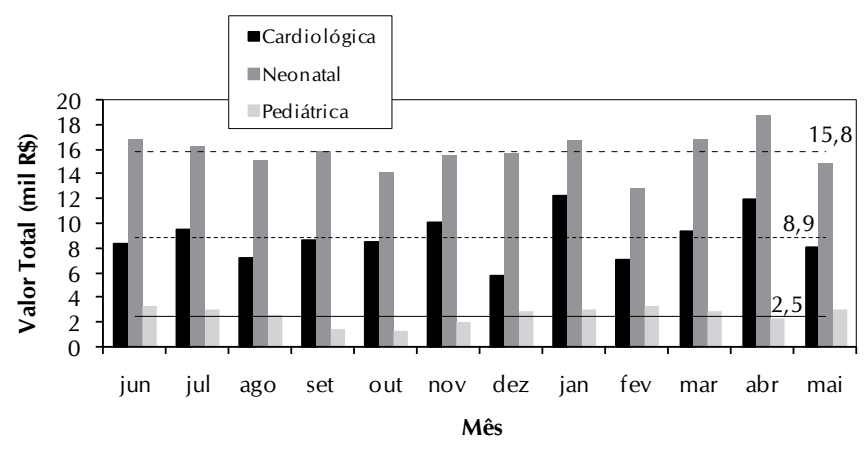

Figura 2 - Histórico mensal por unidades dos gastos com materiais $/ \mathrm{n}^{\circ}$ de leitos ocupados. São José do Rio Preto, 2007 a 2008.

Na Figura 2 pode-se verificar que a média dos gastos mensais com materiais foi de (R\$0,92 $\pm 0,26)$ mil/leito mês - UTI e USI Cardiológica, $(R \$ 0,83 \pm 0,27) \mathrm{mil} /$ leito mês na Neonatal e $(R \$ 0,26 \pm 0,06)$ mil/leito mês - Pediátrica. Esses achados evidenciam que mesmo com menor taxa de ocupação, a UTI cardiológica apresentou o maior gasto com material, por ser de mais alta complexidade.

Nota-se também que houve significativa variação do gasto mensal dessas unidades no período de coleta dos dados, havendo proximidade entre os gastos médios mensais das unidades Cardiológica e Neonatal, sendo esses superiores em até seis vezes ao gasto médio das UTI e USI Pediátrica. Também, o gasto total/mês e gasto/leito/mês, bem como, os tipos e custos de materiais utilizados evidenciaram a menor complexidade assistencial na UTI pediátrica quando comparados ao da UTI cardiológica.

O gerenciamento adequado de materiais, desde a fase do planejamento até o consumo pelos clientes, visa manter o equilíbrio entre os recursos disponíveis e a qualidade da assistência prestada. Para isso, pode-se utilizar como ferramenta a implementação de um sistema de custeio, permitindo uma análise diferenciada dos dados.

Por fim, vale aprofundar a discussão quanto à utilização da classificação da curva $\mathrm{ABC}$ na análise dos dados deste estudo.

\section{Classificação da curva $\mathrm{ABC}$}

$O$ método $A B C$ se revelou como um importante instrumento para utilização no gerenciamento dos estoques nas organizações, sendo também perfeitamente aplicável na gestão de materiais e insumos médicos ${ }^{(2)}$. Vale esclarecer, inicialmente, que os itens de materiais da Classe B não foram abordados no presente estudo.

Aplicando-se a classificação $\mathrm{ABC}$ aos dados analisados, a Classe $\mathrm{A}$ se compôs pelos dez materiais de maior custo (Tabela 2) das UTI e USI Cardiológica e Neonatal, que corresponderam a 24,2 e $15 \%$ dos itens consumidos, e a $40 \%$ e $47 \%$ do orçamento dessas unidades, respectivamente. Os materiais hospitalares incluídos na Classe A são importantes, por representarem um grande investimento, exigindo assim um controle minucioso e frequente e tem como objetivo a redução dos prazos de abastecimento, dos estoques de reserva, o estabelecimento de controles de utilização e a busca de melhores preços ${ }^{(2-3)}$.

A equipe de enfermagem tem papel fundamental no uso eficiente dos recursos materiais em instituições de saúde, desenvolvendo um olhar crítico sobre o processo de trabalho por meio da conscientização. Estudo realizado em hospital universitário constatou -se que os materiais pertencentes à Classe A , 67(31,1\%) eram materiais assistenciais, 80\% utilizados pela equipe de enfermagem ${ }^{(3)}$. Já em pesquisa em UTI neonatal, demonstrou gastos por uso indevido/irracional de materiais num total de $\mathrm{R} \$ 7.208,95$, representando impacto no orçamento hospitalar ${ }^{(23)}$.

Pela Tabela 1 verifica-se que, na Classe $C$, enquadraram-se os oito materiais mais consumidos nas UTI e USI cardiológica, neonatal e pediátrica, correspondendo a 72,5\%, 65,9\% e $79,2 \%$ dos itens consumidos e a $14,4 \%, 10,7 \%$ e $45,8 \%$ do orçamento dessas unidades, respectivamente. Com os itens da Classe $C$ pode-se trabalhar com maiores prazos de abastecimento, aumentar os estoques de reserva e os controles podem ser mais flexíveis ${ }^{(2)}$. No hospital campo desta pesquisa os materiais da Classe $\mathrm{C}$ estavam disponíveis nas unidades estudadas em sub-estoques, dificultando seu controle no aspecto quantitativo e prazo de validade, especialmente, uma vez que eram solicitados para a unidade e não para cada paciente.

É essencial conhecer os custos dos serviços de saúde, já que possibilita a identificação das unidades que necessitam reduzí-los, controlar os gastos e eliminar os desperdícios ${ }^{(1)}$. $\mathrm{Na}$ área da saúde é preciso manter o foco no aumento da qualidade dos cuidados e, ao mesmo tempo, propor e implementar sistemas de gerenciamento de custos, visando a contenção de gastos $^{(3-5)}$. Para a criação, implementação e controle desse sistema de gestão envolve a participação da área administrativa e dos gerentes das diferentes unidades que compõem uma organização hospitalar, de forma que cada integrante contribua com seu conhecimento específico ${ }^{(3)}$.

\section{CONCLUSÃO}

Os achados desta investigação permitiram concluir que a classificação ABC propicia parâmetros para a gestão de custos em unidades pediátricas de terapia intensiva e semi-intensivas, 
no que se refere à maximização dos recursos materiais e redução dos desperdícios. Portanto, para delinear o consumo e gastos de materiais de unidades complexas de atendimento em saúde, o enfermeiro tem muito a contribuir, desde que conheça o perfil da sua clientela, o tipo assistencial e a abordagem terapêutica necessária. Este delineamento pode auxiliar o enfermeiro em UTI e USI pediátricas no planejamento, controle e avaliação na gestão de recursos materiais.

\section{REFERÊNCIAS}

1. Kurcgant P, Coordenadora. Gerenciamento em Enfermagem. Rio de Janeiro: Guanabara Koogan; 2012.

2. Vecina Neto G, Ferreira Júnior WC. Administração de materiais para sistemas locais de saúde. In: Escola Politécnica de Saúde Joaquim Venâncio. Administração. Rio de Janeiro: Fiocruz, 2001. p. 117-58.

3. Lourenço KG, Castilho V. Classificação $A B C$ dos materiais: uma ferramenta gerencial de custos em enfermagem. Rev Bras Enferm 2006;59(1):52-5.

4. Dallora MELV, Foster AC. A importância da gestão de custos em hospitais de ensino - considerações teóricas. Medicina (Ribeirão Preto) 2008;41(2):135-42.

5. Junior AP. Gerenciamento de recursos materiais em unidades de saúde. Rev Espaço Saúde 2005;7(1): 30-45.

6. Martha VF, Garcia PC, Piva JP, Einloft PR, Bruno F, Rampon $\mathrm{V}$. Comparação entre dois escores de prognóstico (PRISM e PIM) em unidade de terapia intensiva pediátrica. J Pediatr (Rio J) 2005;81:259-64.

7. Paterno D. Administração de materiais São Paulo. Monografia [Graduação]. Universidade São Camilo; 1985.

8. Ministério da Saúde (Brasil). Resolução RDC $n^{\circ}$. 07, de 24 de fevereiro de 2010. Dispõe sobre os requisitos mínimos para funcionamento de Unidades de Terapia Intensiva e dá outras providências. Diário Oficial da União 25 fev 2010.

9. WB, Shin SH, Cordeiro AMG. Disponibilidade de unidades de terapia intensiva pediátrica e neonatal no município de São Paulo. J Pediatr (Rio J) 2004;80:453-60.

10. Hatem TP, Lira PI, Mattos SS. The therapeutic effects of music in children following cardiac surgery. J Pediatr (Rio J) 2006;82:186-92.

11. Sakita NK. Cateterismo central por inserção periférica em UTI neonatal de nível terciário: incidência de complicações e fatores de risco associados. São Paulo. Dissertação [Mestrado em Enfermagem]- Universidade de São Paulo; 2009.

12. Araújo TE. Prevalência do uso de profilaxia para sangramento digestivo relacionado ao estresse em pacientes internados em UTI pediátricas de cinco hospitais de Porto Alegre. Porto Alegre. Dissertação [Mestrado em Ciências Médicas]- Universidade Federal do Rio Grande do Sul; 2009.
13. Conselho Federal de Enfermagem (Brasil). Resolução COFEN No 293/2004. Fixa e Estabelece Parâmetros para o Dimensionamento do Quadro de Profissionais de Enfermagem nas Unidades Assistenciais das Instituições de Saúde e Assemelhados [resolução na internet]. [acesso em 6 set 2009]. Disponível em < http://www.saude.mg.gov. br/atos normativos/legislacao-sanitaria/estabelecimentosde-saude/exercicio-profissional/res_293.pdf >

14. Ministério da Saúde (Brasil). Portaria MS no ${ }^{\circ} 1071$ de 4 de julho de 2005. Política Nacional de Atenção ao Paciente Crítico. [portaria na internet]. Diário Oficial da União 8 jul 2005. [acesso em 2 dez 2009]. Disponível em: <http:// www.sobrati.com.br/ms-politica-critico.htm >

15. Perroca MG, Jericó MC, Calil ASG. Composição da equipe de enfermagem em Unidades de Terapia Intensiva. Acta Paul Enferm 2011;24(2):199-205

16. Weinstein SM. Principles and practice of intravenous therapy. 8. ed. Philadelphia: Lippincott Williams \& Wilkins, 2007.

17. Silva AEBC. Análise de risco do processo de administração de medicamentos por via intravenosa em pacientes de um hospital universitário de Goiás. Ribeirão Preto. Tese [Doutorado em Enfermagem]- Escola de Enfermagem de Ribeirão Preto da Universidade de São Paulo; 2008.

18. Zuliani LL,Jericó MC. Estudo comparativo do consumo e gasto com medicamentos em Unidades Pediátricas Intensiva e Semi-intensiva. Rev Paul Pediatr 2012;30:107-15.

19. Souza FI, Teske M, Sarni RO. Parenteral nutrition in preterm infants: proposal of a practical guideline. Rev Paul Pediatr 2008;26:278-89.

20. Harada MJCS, Pedreira MLG. Terapia intravenosa e infusões.São CaetanoSul: Yendis; 2011.

21. Conselho Federal de Enfermagem (Brasil). Resolução $\mathrm{n}^{\circ}$. 258, de 12 de julho de 2001. Inserção de cateter periférico central pelos enfermeiros.

22. Magalhães FJ, Lima FET, Rolim KMC, Cardoso MVLML, Sherlock MSM, Albuquerque NLS. Respostas fisiológicas e comportamentais de recém-nascidos durante o manuseio em unidade de terapia intensiva neonatal. REME Rev Min Enferm 2011;12(1):136-43.

23. Lopes LA, Dyniewicz AM, Kalinowski LC. Gerenciamento de materiais e custos hospitalares em UTI neonatal. Cogitare Enferm 2010;15(2):278-85. 